\title{
FLAT RIEMANNIAN MANIFOLDS
}

\author{
ALPHONSE T. VASQUEZ
}

Some years ago, Auslander and Szczarba [2] gave an example of a compact flat manifold having nontrivial Stiefel-Whitney classes. This was, I imagine, somewhat surprising in view of the fact that the (real) Pontryagin classes of any Riemannian manifold can always be expressed in terms of the curvature tensor and thus are trivial for flat manifolds. $\S 5$ of this paper is devoted to more examples of this general sort-the general paucity of examples in this area being a great hindrance to any reasonable conjectures. The only difficulty here is finding nontrivial yet computable situations.

The Main Theorem 2.3 of this paper is a decomposition theorem of sorts for compact flat Riemannian manifolds, and has strong consequences for the study of Stiefel-Whitney classes of such manifolds. The theorem is in the same general spirit as that of Calabi [17], but seems technically unrelated.

Perhaps the simplest compact flat manifolds are flat tori-these are the ones with trivial holonomy group. In $\S 2$ we describe the notion of a "flat toral extensions". Roughly speaking, this is a way of putting together one compact flat manifold and a flat torus to make a new flat manifold the dimension of which is the sum of the dimensions of its constituents. It is, more technically speaking, a fiber bundle over the manifold with a flat torus as fiber. The group of the bundle is a quotient of the holonomy group of the manifold and it acts on the flat torus isometrically. The tangent bundle of such a construct turns out to be induced from a bundle over the base space-a technical fact which we exploit to obtain information about characteristic classes of flat manifolds.

The main theorem states that under some conditions a compact flat manifold arises as a flat toral extension. The condition is stated in terms of the holonomy group of the manifold: to wit

There is associated with each finite group $\Phi$ a positive integer $n(\Phi)$ such that: if $M$ is a compact flat manifold with holonomy group $\Phi$, and $\operatorname{dim} M>$ $n(\Phi)$, then $M$ is a flat toral extension of another flat manifold of dimension $\leq n(\Phi)$.

It is convenient to say $M$ is a $\Phi$-manifold if it is compact, flat and its holonomy group is isomorphic to $\Phi$. The theorem implies that all $\Phi$-manifolds "are" flat toral extensions of a finite set of flat manifolds. Here "are" means

Communicated by R. S. Palais, September 25,1969 . This paper was partially supported by NSF grant GP-7694. 
up to connection preserving diffeomorphism (not, as might be expected, isometry). Among the consequences of this fact we mention the following. The characteristic algebra of a $\Phi$-manifold vanishes in dimensions $>n(\Phi)$, (2.8). If $\operatorname{dim} M>n(\Phi)$, then $M$ is a cobordant to zero and hence only finitely many $\Phi$-manifolds can fail to bound, (2.4).

We note that it is an open question whether or not all flat manifolds bound. A mild variant of Theorem 3.6 was first conjectured by L. Auslander, in one of many stimulating and fruitful conversations on this and related topics.

Notation and conventions. $\quad R^{n}$ will denote Euclidean space of dimension $n$ considered as a Riemannian manifold with its usual metric. A full-lattice $L$ of $R^{n}$ is a subgroup of $R^{n}$, which is generated by $n$ linearly independent elements of $R^{n}$. The quotient space $R^{n} / L$ is thus an $n$-torus-i.e., a manifold diffeomorphic to a product of $n$ circles. A flat-torus is an $R^{n} / L, L$ a full-lattice, considered as a Riemannian manifold, with the induced Riemannian metric; it is a flat in the classical sense of having zero curvature.

A manifold will mean a connected differentiable manifold of class $C^{\infty}$. The tangent bundle $\tau$ (or $\tau(M)$ ) of a manifold $M$ is, of course, an $n$-dimensional real vector bundle over $M$. As such (at least when $M$ is paracompact) it can be induced from the universal $n$-dimensional vector bundle $\zeta_{n}$ over the space $B_{0(n)}$, the classifying space for the orthogonal group $O(n)$. See [11] for this notion. Indeed, there is a well-defined homotopy class $[\tau]$ of maps from $M$ to $\boldsymbol{B}_{0(n)}$ which "classify" $\tau$; i.e., $f: M \rightarrow B_{0(n)}$ is in $[\tau]$ if and only if $f^{*}\left(\zeta_{n}\right)$ is equivalent to $\tau$. Since all the $f^{\prime}$ s which classify $\tau$ are homotopic, they all induce the same homomorphism $H^{*}\left(B_{0(n)} ; R\right) \rightarrow H^{*}(M ; R)$. The image of this map is the characteristic subalgebra of $M$; any element in the image, a characteristic class of $M$. Here $R$ can be any ring of coefficients.

For example, for $R=Z_{2}=$ the field of two elements, $H^{*}\left(B_{0(n)}, Z_{2}\right)$ $\simeq Z_{2}\left[w_{1}, \cdots, w_{n}\right]$ for some $w_{i} \in H^{i}\left(B_{0(n)}, Z_{2}\right), i=1, \cdots, n$, called the universal Stiefel-Whitney classes. The image of $w_{i}$ in $H^{i}\left(M, Z_{2}\right)$ via any classifying map is the $i$-th Stiefel-Whitney class of the manifold $M$. Similarly for $R=Z$, the group of integers, there are universal Pontryagin classes $p_{i} \in H^{4 i}\left(B_{0(n)}, Z\right)$; and corresponding $p_{i}(M) \in H^{4 i}(M ; Z)$, Pontryagin classes for the manifold $M$.

If $M$ is a compact $n$-manifold $H_{n}\left(M, Z_{2}\right) \simeq Z_{2}$, the non-zero element is the fundamental class of $M$ (denoted $[M])$. If $i_{2} \leq \cdots \leq i_{k}$ and $i_{1}+\cdots+i_{k}$ $=n$, then $w_{i_{1}}(M) \cdots w_{i_{k}}(M) \in H^{n}\left(M ; Z_{2}\right) \simeq \operatorname{Hom}\left(H_{n}\left(M, Z_{2}\right), Z_{2}\right)$. The result of evaluating this cohomology class on the fundamental class yields an element of $Z_{2}$ (notation $w_{i_{1}} \cdots w_{i_{k}}[M]$ ); this is the Stiefel-Whitney number of $M$ corresponding to the partition $\left(i_{1}, \cdots, i_{k}\right)$ of $n$.

We have a similar notion of Pontryagin number. These are defined where we have $n=4\left(i_{1}+\cdots+i_{k}\right)$ and when $M$ is an oriented $n$-manifold-i.e., a generator $[M] \in H_{n}(M ; Z) \simeq Z$ has been specified. 


\section{General considerations}

Let $M$ be a compact, flat Riemannian manifold. As is well known, its universal covering space can be identified as a Riemannian manifold with $R^{n}$. Consequently, the fundamental group $\pi$ of $M$ acts via isometries of $R^{n}$ in such a way that we may identify $M$ with the space of orbits of this action; symbolically $M=R^{n} / \pi$. The group $\mathscr{E}_{n}$ of all isometries of $R^{n}$ contains a normal subgroup consisting of all translations. This subgroup will be identified in the usual way with $R^{n}$. Denoting by $D$, the natural homomorphism of $\mathscr{E}_{n}$ onto $O(n)=$ the group of orthogonal linear transformations of $R^{n}$ we have $R^{n}=$ Kernel $D$ and thus $D(\pi) \cong \pi / \pi \cap R^{n}$. It is known [3] that this is a finite subgroup of $O(n)$ and is called the holonomy group of $M$. We will use the notation $\Phi=D(\pi)$.

Recall that for any reasonable group $G$, we have the notion of a universal principal $G$-bundle. The total space of such an object we will denote by $E_{G}$; the base space by $B_{G}$. We recall the criterion of [11] which asserts that a principal $G$-bundle is universal if its total space has no non-trivial homotopy. Our first remark is that we have one of these objects at hand; to wit, $R^{n}$ is the total space of a principal $\pi$-bundle with base space $M$; thus $\mathrm{E}_{\pi}=R^{n}$ and $B_{\pi}=M$.

Now $G \rightarrow B_{G}$ behaves more or less like a functor, and in particular, from the surjection $D: \pi \rightarrow D(\pi)=\Phi$ we can cook up a corresponding map $B_{D}: B_{\pi}$ $\rightarrow B_{\Phi}$. Finally, the inclusion $i: \Phi \subset 0(n)$ yields a map $B_{i}: B_{\Phi} \rightarrow B_{0(n)}$. The universal $n$-dimensional vector bundle over $B_{0(n)}$ of course yields, via this map, a vector bundle $\eta$ over $\boldsymbol{B}_{\boldsymbol{p}}$.

Proposition 1.1. $B_{D}^{*}(\eta)$ is equivalent to the tangent bundle of $M$.

Proof. We have a commutative diagram as follows

$$
\begin{array}{r}
R^{n}=E_{\pi} \stackrel{E_{D}}{\longrightarrow} E_{\Phi} \\
\downarrow \\
\downarrow=R^{n} / \pi=B_{\pi} \stackrel{B_{D}}{\longrightarrow} B_{\Phi}
\end{array}
$$

where $E_{D}(\sigma \cdot e)=D(\sigma) \cdot E_{D}(e)$ for all $\sigma \in \pi$ and $e \in E_{\pi}=R^{n}$.

Let the total space of $\eta$ be $E_{\emptyset} \times R^{n} / \Phi$ where $g \in \Phi$ acts via $g(e, v)=(g \cdot e$, $g \cdot v)$. Now clearly the total space $\tau$ of the tangent bundle of $M=B \Phi$ can be taken to be $R^{n} \times R^{n} / \pi$ where $\pi$ acts via

$$
\sigma\left(v_{1}, v_{2}\right)=\left(\sigma v_{1}, D(\sigma) v_{2}\right)
$$

Thus we clearly have a commutative diagram as follows:

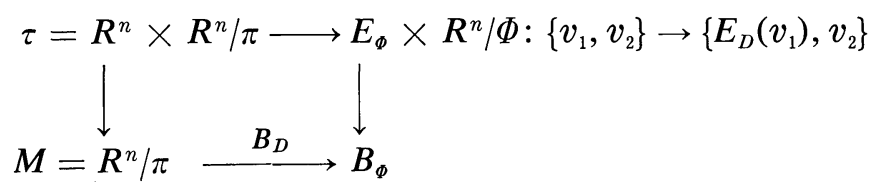


and the result follows.

Now it follows that $B_{i} \circ B_{D}: B_{\pi}=M \rightarrow B_{0(n)}$ is the map which classifies the tangent bundle of $M$. The following conclusions are elementary.

Corollary 1.2. If $a \in H^{i}(M, R), i>0$, is in the characteristic algebra of $M$, then $N a=0$ where $N=$ the order of $\Phi$.

Proof. Since the map in question factors through $B_{\phi}$, this is a consequence of the fact that $H^{i}\left(B_{\Phi} ; R\right) \cong H^{i}(\Phi ; R)$ and it is known that every element $b \in H^{i}(\Phi ; A)(i>0)$ satisfies $N b=0$; see [4].

Corollary 1.3. If the holonomy group of $M$ has odd order, all its StiefelWhitney classes $w_{i}$ are zero $(i>0)$. Consequently all its Stiefel-Whitney numbers vanish and it is therefore the boundary of some compact smooth manifold of dimension $n+1$.

Proof. The first few statement is an immediate consequence of Corollary 1.2 and the rest follows from the results of Thom [12].

Corollary 1.4. The Pontryagin classes $p_{i}(M) \in H^{4 i}(M ; Z)$ of $M$ are torsion classes; and consequently if $M$ is orientable, its Pontryagin numbers are all zero.

Proof. Again a trivial consequence of Corollary 1.2. Alternatively a proof can be made from the fact that the image of $p_{i}(M)$ in $H^{4 i}(M ; R)(R=$ the real numbers) can be represented explicitly in terms of the curvature tensor which is 0 since $M$ is flat.

Corollary 1.5. If the holonomy group of $M$ has odd order, it is necessarily orientable and is the oriented boundary of a compact oriented smooth $(n+1)$ - manifold.

Proof. Since $w_{1}(M)=0$ (Cor. 1) $M$ is orientable and now the result is a trivial consequence of the knowledge that the Stiefel-Whitney and Pontryagin numbers are complete invariants of oriented cobordism. See [12] and [14].

Remark. It is known [2] that $w_{i}(M)$ need not always be 0 . The holonomy group of the relevant $M$ is $Z_{2} \times Z_{2}$. Just what can be said about the characteristic algebra of a flat manifold seems a very difficult question. In the last section of the paper, we give more examples in the spirit of [2] where the Stiefel-Whitney classes turn out to be non-trivial. On the other hand, one consequence of the theorems below is that the characteristic algebra of a flat manifold vanishes above a dimension which depends 'only on the holonomy group.

\section{Flat toral extensions}

If $M$ is a manifold and $m_{0} \in M$, we know that $\pi=\pi_{1}\left(M, m_{0}\right)$ acts as a group of diffeomorphisms on $\bar{M}$, the universal covering space of $M$. Further $M$ can be identified as the space of orbits of this action-a situation we symbolize by $M=\grave{M} / \pi$.

Suppose now that $\pi$ also acts as a group of diffeomorphisms of a manifold 
$F$. We can let $\pi$ act diagonally on $\bar{M} \times F-$ i.e., $g \in \pi, \tilde{m} \in M, f \in F$, $g \cdot(\tilde{m}, f)=(g \cdot \tilde{m}, g \cdot f)$, and form the space of orbits $\bar{M} \times F / \pi$. There is an obvious map $\rho: \bar{M} \times F / \pi \rightarrow \bar{M} / \pi=M$, and it is readily verified that we have here a fiber bundle in the sense of Steenrod. The fiber, of course, is $F$ and the group is $\pi$ (with the discrete topology!). The total space can be given a smooth structure in an obvious way and $\rho$ is smooth.

If $M$ and $F$ are both Riemannian manifolds, and if $\pi$ acts via isometries on $F$, then (with the obvious Riemannian structure on $\bar{M}$ ) $\pi$ acts isometrically on $\bar{M} \times F$ and yields a Riemannian structure on $\bar{M} \times F / \pi$. Clearly this process is such that if both $M$ and $F$ are flat, then $\bar{M} \times F / \pi$ is also flat.

Definition 2.1. A flat-toral extension of the Riemannian manifold $M$ is any Riemannian manifold isometric to $\bar{M} \times F / \pi$ where $F$ is a flat torus on which $\pi$ acts via isometries. It is convenient to adopt the convention that a single point is a 0 -dimensional flat torus. If this is done, $M$ is itself among the flat toral extensions of $M$. The others will be called non-trivial flat toral extensions of $M$.

The following proposition follows readily from the definition but we will defer its proof until later so that we can state the main theorem and one of its corollaries.

Proposition 2.2. A compact non-trivial flat toral extension is always a boundary.

Following [6], we call $M$ a $\Phi$-manifold if its holonomy group is isomorphic to the group $\Phi$.

Main Theorem 2.3. Let $\Phi$ be a finite group. Then there is an integer $n(\Phi)$ such that: if $M$ is any compact flat-Riemannian manifold with holonomy group isomorphic to $\Phi$, then $M$ is a flat toral extension of some compact flat Riemannian manifold of dimension $\leq n(\Phi)$.

We note that the theorem is trivially true for $M$ 's such that $\operatorname{dim} M \leq n(\Phi)$ for such an $M$ is a trivial flat toral extension of itself.

Corollary 2.4. All $\Phi$-manifolds of dimension $>n(\Phi)$ are boundaries.

Since up to affine (not necessarily Riemannian) equivalence, there are only finitely many flat manifolds in each dimension we conclude: only finitely many affine equivalence classes of $\Phi$-manifolds can fail to bound.

Remark 2.5. Very little is known about $n(\Phi)$. The proof yields $n(\Phi)=1$ if $\Phi$ has prime order and very little more. The final section shows by calculation of some special cases that $n\left(Z_{2}^{2 k}\right) \geq 2 k$ and that $n\left(Z_{2}^{2 k+1}\right) \geq 2 k$.

We turn to some preliminaries to the proof of the proposition. The basic business is to examine the tangent bundle along the fibers. The fiber of this bundle at a point $e$ is the kernel of the surjection $d \rho: T(E)_{e} \rightarrow T(B)_{\rho(e)}$.

Lemma 2.6. If $M$ is a Riemannian manifold, and $M^{\prime}=\bar{M} \times F / \pi$ is a non-trivial flat toral extension of $M$, then the tangent bundle along the fibers is induced from a vector bundle over $M$ via the projection map.

Proof. $F=R^{n} / L$ for some lattice $L . \quad R^{n}$ is clearly its universal covering 
space. If $\sigma: F \rightarrow F$ is any diffeomorphism, it lifts to a diffeomorphism $\tilde{\sigma}: R^{n}$ $\rightarrow R^{n}$. $\tilde{\sigma}$ is determined not uniquely but up to a translation by an element of $L$. It follows that the differential $D(\tilde{\sigma})(x): R^{n} \rightarrow R^{n}$ depends only on $p(x) \in F$ where $p$ is the canonical projection. We may thus denote it by $D(\sigma)_{y}, y=p(x)$. It is trivial that $D\left(\sigma_{1} \circ \sigma_{2}\right)_{y}=D\left(\sigma_{1}\right)_{\sigma_{2}(y)} \circ D\left(\sigma_{2}\right)_{y}$. Consequently it is readily verified that if $\pi$ acts via diffeomorphisms, then the tangent bundle along the fibers is the space $\bar{M} \times F \times R^{n} / \pi$ where

$$
\sigma \cdot(\tilde{m}, y, v)=\left(\sigma \cdot \tilde{m}, \sigma(y), D(\sigma)_{y} v\right) .
$$

Now, however, if $\pi$ acts via isometries of $F$, then each $\tilde{\sigma}$ is an affine map and $D(\tilde{\sigma})_{x}$ is wholly independent of $x$. Thus we may form

$$
\tilde{M} \times R^{n} / \pi \quad \text { by } \quad \sigma(\tilde{m}, v)=\left(\sigma \tilde{m}, D(\sigma)_{x}(v)\right)
$$

for any $x$. This is a vector bundle over $\bar{M} / \pi=M$ and the obvious projection map $\bar{M} \times F / \pi \rightarrow \bar{M} / \pi=M$ is covered by a bundle map $\bar{M} \times F \times R^{n} / \pi \rightarrow$ $\bar{M} \times R^{n} / \pi$.

Lemma 2.7. The characteristic algebra of $M^{\prime}$ vanishes in dimensions exceeding the dimension of $M$.

Proof. If $\rho: M^{\prime} \rightarrow M$ is the projection, we have the usual exact sequence $0 \rightarrow$ bundle along the fibers

$\rightarrow$ tangent bundle of $M^{\prime} \rightarrow \rho^{-1}$ (tangent bundle of $\left.M\right) \rightarrow 0$.

Since $M^{\prime}$ is Riemannian, it follows that $T\left(M^{\prime}\right)$ is the Whitney sum of the two extreme bundles and consequently $T\left(M^{\prime}\right)=\rho^{-1}$ (a bundle over $M$ ). Thus the map which classifies the tangent bundle of $M^{\prime}$ factors through $M$. Since $H^{i}(M)$ $=0$ for $i>\operatorname{dim} M$, we are done.

The proposition trivially follows from Lemma 2.7 , since a manifold bounds if and only if all its Stiefel-Whitney numbers vanish. See [12].

Corollary 2.8. Suppose $M$ is a compact flat Riemannian manifold with holonomy group $\Phi$. Then the characteristic algebra of $M$ vanishes in dimensions greater than $n(\Phi)$.

Same proof.

\section{The basic algebraic theorem}

A compact flat Riemannian manifold is "determined" in a strong sense by its fundamental group, and so we formulate and prove here some algebraic facts which are more or less equivalent to the main theorem.

The first order of business is to be able to recognize the fundamental group of a compact flat Riemannian manifold. The basic result here is in [1]. We want a mild looking variant of this theorem. It seems annoyingly difficult to prove. Indeed, an imitation of the proof in [1] would really be about as efficient as the path taken here; but there is a mildly amusing algebraic lemma along the way. 
Theorem 3.1. $\pi$ is the fundamental group of a compact flat Riemannian manifold of dimension $n$ if and only if

a) $\pi$ is torsion free,

b) $\pi$ contains a normal subgroup $N$ which is free abelian of rank $n$ and, furthermore,

c) $\pi / N$ is a finite group.

Proof. We reduce this theorem to a completely analogous one in [1]. The only difference between the conditions here and there is that in [1] it is required in addition that $N$ be maximal abelian. Thus the burden of our proof is to show that the existence of $N$ implies the existence of an $N^{\prime}$ which is normal, maximal abelian, free abelian of rank $n$ and of finite index. We claim that $N^{\prime}=\{\sigma \in \pi \mid \sigma n=n \sigma \forall n \in N\}$ is such a group. It is trivially normal and if abelian, clearly maximal abelian. Indeed, the only difficult statement is that $N^{\prime}$ is abelian. We defer this for a moment and show that if abelian $N^{\prime}$ is free abelian of rank $n$. Since $N^{\prime} / N \subset \pi / N, N^{\prime} / N$ is finite and so $N^{\prime}$ is finitely generated; and since $\pi$ is torsion free, it must be free abelian of some rank. But again, since $N^{\prime} / N$ is finite, this rank must be $n$. That $N^{\prime}$ is abelian is a trivial consequence of the following lemma.

Lemma 3.2. A torsion-free central extension of a finite group is necessarily abelian.

Proof. Suppose $\varphi: G \rightarrow \Phi$ is a homomorphism onto the finite group $\Phi$ with Kernel $\varphi \subset$ Center $(G)$ and $G$ torsion-free. Calling $K=$ Kernel $\varphi$ we have (see [8]) an exact sequence as follows:

$$
H_{2}\left(\Phi, H_{0}(K, Z)\right) \rightarrow H_{0}\left(\Phi ; H_{1}(K, Z)\right) \rightarrow H_{1}(G, Z) \rightarrow H_{1}(\Phi, Z) \rightarrow 0 .
$$

Since $K$ is central, $K$ is a trivial $\Phi$ module so the second group "is" $H_{1}(K, Z)$; and since $K$ is abelian this in turn "is" $K$. But since $\Phi$ is finite, every element of the first group is of finite order; and since $K$ is torsion-free, the first homomorphism must be trivial. Replacing $H_{1}(G, Z)$ by $G /[G, G]$ we thus have the following exact sequence:

$$
0 \rightarrow K \rightarrow G /[G, G] \rightarrow H_{1}(\Phi, Z) \rightarrow 0 .
$$

The morphism from $K$ to $G /[G, G]$ is induced by the inclusion $K \subset G$, and thus $K \cap[G, G]=\{1\}$, or equivalently $[G, G]$ is isomorphic to a subgroup $G / K=\Phi$, a finite group. Thus $[G, G]$ is a finite subgroup of the torsion-free $G$ and hence $[G, G]=\{1\}$.

Remarks 3.3. The group $N^{\prime}$ being maximal abelian and of finite index in $\pi$ is easily shown to consist precisely of those elements of $\pi$ having only finitely many conjugates. This canonically determined subgroup we will denote by $t(\pi)$, and call it the translation subgroup of $\pi$. Clearly the finite group $\pi / t(\pi)$ acts faithfully on $t(\pi)$, and is called the holonomy group of $\pi$. We will call rank $(t(\pi))$ the dimension of $\pi$. It is convenient to introduce some notation. 
Definition 3.4. A group is a Bieberbach group if and only if it contains a normal, free abelian, finitely generated subgroup of finite index. Thus the class of torsion-free Bieberbach groups is precisely the class of groups we have just been discussing. We can summarize all this in a convenient package.

Theorem 3.5. A group $\pi$ is the fundamental group of an $n$ dimensional compact flat Riemannian manifold if and only if it is a torsion-free Bieberbach group of dimension $n$. The subset $t(\pi)$ consisting of elements having finitely many conjugates is a maximal abelian normal subgroup of finite index and is a free abelian group of rank $n$. The homological dimension of such a group is, in fact, $n$.

Proof. All but the last statement has already been proved. To do this let $M$ be a compact flat Riemannian $n$-manifold with fundamental group $\pi$. According to [5], this space can be triangulated as a finite simplicial complex. Its universal covering space $\bar{M}$ can therefore be compatibly triangulated. Let $C_{i}$ be the group of oriented simplicial chains of $\bar{M}$. Since $\pi$ acts as a group of deck transformations on $\bar{M}$, and the triangulation of $\bar{M}$ is compatible with that of $M$, it is easy to verify that $C_{i}$ is in an obvious way a free $Z[\pi]$ module with one generator for each oriented $i$-simplex of the triangulation of $M$. Since $M$ is an $n$-dimensional manifold, $C_{i}=0$ for $i>n$. Also since $\bar{M}$ can be identified as a Riemannian manifold with $R^{n}$, we know that the complex $\left(C_{*}, \partial_{*}\right)$ is acyclic. Thus we have exhibited a resolution of length $n$ of the trivial $Z[\pi]$-module $Z$ by free $Z[\pi]$-modules. It follows that the homological dimension of $\pi$ is less than or equal to $n$. To see that it is exactly $n$ we note that if we view $Z_{2}$ as a trivial $\pi$-module, then $C_{i} \otimes_{\pi} Z_{2}$ is canonically isomorphic to the simplicial chain group of $M$ with coefficients $Z_{2}$. Since $M$ is a compact, connected $n$-manifold, we know $H_{n}\left(C_{*} \otimes Z_{2}\right) \simeq Z_{2}$, and hence $H_{n}\left(\pi ; Z_{2}\right)$ $=Z_{2}$ and the homological dimension of $\pi$ is at least $n$.

The following is the main algebraic theorem in the paper. See [13] where it was announced.

Theorem 3.6. Let $\Phi$ be a finite group. Then there is an integer $n(\Phi)$ such that: if $\pi$ is a torsion-free Bieberbach group with holonomy group isomorphic to $\Phi$, then $t(\pi)$ contains a normal subgroup $A^{\prime}$ such that $\pi / A^{\prime}$ is a torsion-free Bieberbach group of dimension $\leq n(\Phi)$.

We remark here that the difficulty lies in controlling torsion in $\pi / A^{\prime}$. Before proceeding with the proof we mention the machinery used in keeping track of the torsion. Associated with the hypothesis of Theorem 3.6 we have the exact sequence

$$
0 \longrightarrow N \longrightarrow \pi \stackrel{\rho}{\longrightarrow} \Phi \longrightarrow 1 \text {, }
$$

where $N=t(\pi)$ and $\Phi \simeq \pi / t(\pi)$. We use 0 and 1 to emphasize the fact that we will write $N$ additively and $\Phi$ multiplicatively.

If we have any exact sequence such as $\left(^{*}\right)$, we may view $N$ as a module over $\Phi$ via 


$$
\sigma \cdot n=g n g^{-1} \quad \text { for any } g \in \rho^{-1}(\sigma) .
$$

This is well defined since $N$ is abelian. Also the extension itself is described by an element $\alpha \in H^{2}(\Phi, N)$ (for details see [4] and [6]).

Indeed, given the $\Phi$-module $N$, any $\alpha \in H^{2}(\Phi, N)$ will correspond to some exact sequence such as $(*)$. In particular the isomorphism class of the group in the middle of that exact sequence is determined. Calling it $\pi_{\alpha}$ we formulate conditions on $\alpha$ which tell us about torsion in $\pi_{\alpha}$.

Lemma 3.7. Suppose the $\Phi$-module $N$ is a torsion-free group. The extension $\pi_{\alpha}$ has torsion if and only if $J_{\Phi^{\prime}}(\alpha) \in H^{2}\left(\Phi^{\prime}, N\right)$ is zero for some cyclic group $\Phi^{\prime}$ of prime order in $\Phi$. Here $J_{\Phi^{\prime}}: H^{2}(\Phi, N) \rightarrow H^{2}\left(\Phi^{\prime}, N\right)$ is the map induced by the inclusion $\Phi^{\prime} \subset \Phi$.

Proof. If $\pi_{\alpha}$ has torsion, it will have an element $x$ of prime order. Let $\Phi^{\prime}$ be the subgroup of $\Phi$ generated by $\rho(x) \in \Phi$. If $\Phi^{\prime}=\{1\}$, then $x \in N$, which is impossible since $N$ is torsion-free. Thus $\Phi^{\prime}$ is cyclic of prime order. Clearly $J_{\Phi^{\prime}}(\alpha) \in H^{2}\left(\Phi^{\prime}, N\right)$ corresponds to the exact sequence

$$
0 \longrightarrow N \longrightarrow \rho^{-1}\left(\Phi^{\prime}\right) \stackrel{\rho}{\longrightarrow} \Phi^{\prime} \longrightarrow 1 \text {. }
$$

But this sequence clearly splits, i.e., there is a homomorphism $h: \Phi^{\prime} \rightarrow \rho^{-1}\left(\Phi^{\prime}\right)$ such that $\rho \circ h=$ the identity. Indeed let $h(\rho(x))=x$; this works since $x$ has prime order. Hence $J_{\Phi^{\prime}}(\alpha)=0$.

The argument can be reversed to prove the lemma in the other direction.

Remark. It is clear that we could if we wish, restrict ourselves to considering only one $\Phi^{\prime}$ out of each conjugacy class of subgroups of prime order. This remark is conceivably useful in "minimizing" $n(\Phi)$.

Proof of Theorem 3.6. Case I. $\Phi$ is cyclic of prime order. In this case $n(\Phi)=1$ will do. The proof is based on the results of [10] which tell us more than we need to know about the structure of the $\Phi$-module $N$. This paper, indeed, proves that $N$ is the direct sum of various types of sub- $\Phi$-modules. Call the submodules $N_{i}$. Now $N=\oplus N_{i}$ implies $H^{2}(\Phi, N)=\oplus H^{2}\left(\Phi, N_{i}\right)$. A simple argument (see [7] for example where this is done explicitly) shows that each $N_{i}$ is such that either $H^{2}\left(\Phi ; N_{i}\right)=0$ or $N_{i}$ is an infinite cyclic group on which $\Phi$ acts trivially. Since $\alpha \in H^{2}(\Phi, N)$ is not zero, the component of $\alpha$ in one of these $N_{i}$ 's is non-zero. Thus if $A^{\prime}$ is the sum of the other summands, the projection map $N \rightarrow N / A^{\prime}$ takes $\alpha$ into a non-zero element $\alpha^{\prime}$ of $H^{2}\left(\Phi, N / A^{\prime}\right)$. But $\alpha^{\prime}$ corresponds to a torsion-free extension $\pi_{\alpha^{\prime}}$ of $\Phi$ by $N^{\prime} A$. Indeed, one can easily verify that $\pi_{\alpha^{\prime}}$ is itself an infinite cyclic group. In any case $A^{\prime}$ is normal in $\pi$ because $A^{\prime}$ is a $\Phi$-submodule, and clearly $\pi / A^{\prime} \simeq \pi_{\alpha^{\prime}}$ and we are done.

Case II, the general case. For each cyclic subgroup $\Phi^{\prime}$ of prime order, we may view $N$ as a $\Phi^{\prime}$-module. As in Case I there is a $\Phi^{\prime}$-submodule $A^{\prime}\left(\Phi^{\prime}\right)$ of $N$ such that $N / A^{\prime}\left(\Phi^{\prime}\right)$ is an infinite cyclic group and the composite homomorphism 


$$
H^{2}(\Phi, N) \stackrel{J_{\Phi^{\prime}}}{\longrightarrow} H^{2}\left(\Phi^{\prime}, N\right) \longrightarrow H^{2}\left(\Phi^{\prime}, N / A^{\prime}\left(\Phi^{\prime}\right)\right)
$$

is non-trivial on $\alpha$.

Let $A^{\prime \prime}\left(\Phi^{\prime}\right)=\bigcap_{\sigma \in \Phi} \sigma A^{\prime}\left(\Phi^{\prime}\right)$. Clearly $A^{\prime \prime}\left(\Phi^{\prime}\right)$ is a $\Phi$-submodule. Let $A^{\prime}=$ $\cap\left\{A^{\prime \prime}\left(\Phi^{\prime}\right) \mid \Phi^{\prime}\right.$ is a subgroup of prime order $\}$. Clearly $A^{\prime}$ is a $\Phi$-submodule. We now show that $A^{\prime}$ works. The extension $0 \rightarrow N / A^{\prime} \rightarrow \pi / A^{\prime} \rightarrow \Phi \rightarrow 1$ clearly corresponds to the image $\alpha^{\prime}$ of $\alpha$ in $H^{2}\left(\Phi, N / A^{\prime}\right) . \pi / A^{\prime}$ will thus be torsion-free if $\alpha^{\prime}$ never restricts to 0 in $H^{2}\left(\Phi^{\prime} ; N / A^{\prime}\right)$. This follows from the commutativity of the diagram

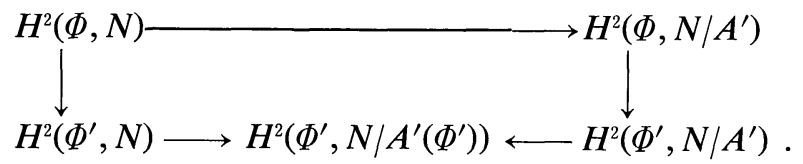

It remains to calculate $\operatorname{dim} \pi / A^{\prime}$ and show that it is bounded by an integer $n(\Phi)$ depending only on $\Phi$.

Clearly $N / A^{\prime} \rightarrow \prod_{\sigma, \Phi^{\prime}} N / \sigma A^{\prime}\left(\Phi^{\prime}\right)$ is an injection. But each factor on the right is a free abelian group of rank 1 and the number of factors depends only on $\Phi$. Thus rank $\left(N / A^{\prime}\right) \leq$ the number of factors on the right. It is clear that $\operatorname{rank}\left(N / A^{\prime}\right)=\operatorname{dim}\left(\pi / A^{\prime}\right)$, although this is not the definition of $\operatorname{dim} \pi / A^{\prime}$.

Corollary 3.8. If $a \in H^{i}(M ; R)$ is in the characteristic algebra of a compact flat Riemannian manifold $M$, and $i>n(\Phi)$ where $\Phi$ is its holonomy group, then $a=0$.

Proof. The classifying map $[\tau]: M \rightarrow B_{0(n)}$ for the tangent bundle of $M$ factors through the map $B_{D}: M=B_{\pi} \rightarrow B_{\Phi}$ where $D: \pi \rightarrow \Phi=\pi / t(\pi)$ is the homomorphism from the fundamental group $\pi$ of $M$ onto the holonomy group $\Phi$. By Theorem 2.4 this homomorphism $D$ factors through a torsion-free Bieberbach group $\pi / A^{\prime}$ where $\operatorname{dim}\left(\pi / A^{\prime}\right) \leq n(\Phi)$. Now by Theorem 3.5 $H^{i}\left(B_{\pi / A^{\prime}}\right)=0$, and hence $H^{i}\left(B_{\pi / A^{\prime}}, R\right)=0$ for $i>\operatorname{dim}\left(\pi / A^{\prime}\right)$, in particular, for $i>n(\Phi)$.

\section{The main theorem}

Our task now is simply translating some algebra into geometry-this will yield flat toral extensions and ultimately, the main theorem, Theorem 1.2.

Theorem 4.1. Suppose $M$ is a compact flat Riemannian manifold with fundamental group $\pi$, and further that $t(\pi)$ contains a normal subgroup $A^{\prime}$ such that $\bar{\pi}=\pi / A^{\prime}$ is torsion-free. Then $M$ is a flat toral extension of a compact flat Riemannian manifold with fundamental group $\bar{\pi}$.

Note. According to Theorem 3.6, such an $A^{\prime}$ will always exist if $\operatorname{dim} M$ $>n(\Phi), \Phi=$ holonomy group of $M$. Since we have already seen (Theorem $3.1)$ that $\bar{\pi}$ is the fundamental group of some compact flat Riemannian manifold, the result comes as no great surprise. 
Proof. We may identify the universal covering space of $M$ as a Riemannian manifold with $R^{n}$, and $\pi$ with a subgroup of the group of Euclidean motions. Letting $D$ : Euclidean motions $\rightarrow$ orthogonal group be the usual homomorphism (indeed it is the derivative) it is clear that Kernel $(D \mid \pi)$ is the subgroup of pure translations and that $D(\pi)$ is the holonomy group of $M$. According to [3], if we identify $R^{n}$ with the group of all translations, then $\operatorname{Ker} D \mid \pi$ is a full lattice. Thus $R^{n}$ is the vector space spanned by $\operatorname{Ker}(D \mid \pi)$. Furthermore, if $\sigma \in \pi$ and $a \in \operatorname{Ker} D \mid \pi \subset R^{n}$, then $\sigma a \sigma^{-1}=D(\sigma)(a)$. It can now be readily verified that $\operatorname{Ker} D \mid \pi$ is the subgroup $t(\pi)$ in the characterization of Theorem 3.5, and that $D(\pi)$ is the holonomy group of $\pi$. Since $A^{\prime}$ is normal in $R^{n}$, if $V^{\prime}$ is the vector space spanned by $A^{\prime}$, then $V^{\prime}$ is $D(\pi)$ invariant. Let $V^{\prime \prime}$ be the orthogonal complement of $V^{\prime}$ in $R^{n}$. Thus $V^{\prime \prime}$ is also $D(\pi)$ invariant. Now it is clear that since $\pi$ is a group of affine transformations of $R^{n}, \pi$ will induce an action on the space of cosets $R^{n} / W$ where $W$ is any $D(\pi)$ invariant subgroup. Thus in particular $\pi$ induces an action on both $R^{n} / V^{\prime}$ and $R^{n} / V^{\prime \prime}$. Indeed the obvious isomorphism $R^{n} \rightarrow R^{n} / V^{\prime} \times R^{n} / V^{\prime \prime}$ is $\pi$-equivariant if we let $\pi$ act diagonally on the right-i.e., simultaneously on both factors. Now $M=R^{n} / \pi$. We calculate this orbit space by calculating on the right; and that calculation we do in two stages. We first let the subgroup $A^{\prime}$ of $\pi$ act and form the orbit space, and then let $\pi / A^{\prime}$ act on the result. Since $A^{\prime} \subset V^{\prime}$, $A^{\prime}$ acts trivially on $R^{n} / V^{\prime}$. Thus $\left(R^{n} / V^{\prime} \times R^{n} / V^{\prime \prime}\right) / A^{\prime}=R^{n} / V^{\prime} \times\left(R^{n} / V^{\prime \prime}\right) / A^{\prime}$. Clearly though $R^{n} / V^{\prime \prime} / A^{\prime}=R^{n} / V^{\prime \prime}+A^{\prime}$, since $A^{\prime}$ is acting on $R^{n}$ by translations. Thus we have $\bar{\pi}$ acting on $R^{n} / V^{\prime} \times R^{n} / V^{\prime \prime}+A^{\prime}$ and the orbit space "is" $M$. To prove the theorem now we want to show that $\bar{\pi}$ is acting on $R^{n} / V^{\prime}$ in such a way that $\left(R^{n} / V^{\prime}\right) / \bar{\pi}$ "is" a compact flat Riemannian manifold and that $R^{n} / V^{\prime \prime}+A^{\prime}$ "is" a flat torus on which $\bar{\pi}$ is acting via isometries. The second fact is easier. Clearly $V^{\prime} \mid A^{\prime} \rightarrow R^{n} / V^{\prime \prime}+A^{\prime}$ is an isomorphism, and if we endow the right hand space with the metric which it inherits from the left, then $\bar{\pi}$ is acting via isometries. We must show that $A^{\prime}$ is a full-lattice in $V^{\prime}$. Since $\pi / A^{\prime}$ is torsion-free, so is $t(A) / A^{\prime}$; therefore we may find free generators $t_{1}, \cdots, t_{n}$ for $t(\pi)$ such that $t_{1}, \cdots, t_{r}$ are free generators for $A^{\prime}$ and the rest yield free generators for $t(A) / A^{\prime}$. Thus $t_{r+1}, \cdots, t_{n}$ must map to a basis in $R^{n} / V^{\prime}$, and $t_{1}, \cdots, t_{r}$ must be a basis for $V^{\prime}$.

It remains then to investigate the action of $\bar{\pi}$ on $R^{n} / V^{\prime}$ and the orbit space. We put a metric on via the isomorphism $V^{\prime \prime} \rightarrow R^{n} / V^{\prime}$ and note that $\bar{\pi}$ is acting as Euclidean motions. Clearly, also, the $t_{r+1}, \cdots, t_{n}$ yield a full-lattice of translations. Thus the map $\varphi: \pi \rightarrow$ Euclidean motions of $\left(V^{\prime \prime}=R^{n} / V^{\prime}\right)$ is clearly one-one on the subgroup $t(\pi) / A^{\prime}$. We will show that this implies that $\varphi$ is one-one on all of $\bar{\pi}$.

Suppose $\sigma \in \pi$ and $\varphi(\{\sigma\})=1$. Since $\pi / t(\pi)$ is finite for some $r \geq 1, \sigma^{r} \in t(\pi)$, hence $\varphi\left(\left\{\sigma^{r}\right\}\right)=1$. But we have just seen that $\varphi$ is one-one on $t(\pi) / A^{\prime}$; so $\sigma^{r} \in A^{\prime}$, and $\{\sigma\}$ is of finite order in $\bar{\pi}$ which is torsion-free by assumption. 
Hence $\{\sigma\}=1$. It now follows from the argument of [1] that $\bar{\pi}$ is acting in such manner that $V^{\prime \prime} / \bar{\pi}$ is a compact manifold.

Remark 4.2. The proof of the main theorem (Theorem 2.3) is simply the juxtaposition of the Theorems 3.6 and 4.1.

\section{Examples}

For each $n \geq 1$, we define subgroups $G_{n}$ of $\varepsilon_{2 n+1}=$ the group of Euclidean motions of $R^{2 n+1}$, which are torsion-free Bieberbach groups imbedded in such a way that $M^{2 n+1}=R^{2 n+1} / G_{n}$ is a compact flat Riemannian manifold. These groups generalize, in an obvious fashion, the example in [2]. We calculate $w_{i}\left(M^{2 n+1}\right)$ and find some non-zero classes. The holonomy group of $M^{2 n+1}$ is $Z_{2} \times \cdots \times Z_{2}=Z_{2}^{n}$ (n-factors). Since $w_{2 i}\left(M^{2 n+1}\right) \neq 0$ for $2 i \leq n$, we can conclude that $n\left(Z_{2}^{2 k}\right) \geq 2 k$ and $n\left(Z_{2}^{2 k+1}\right) \geq 2 k$.

Definition of $G_{n}$. $G_{n}$ is the subgroup generated by $2 n+1$ elements $t_{1}, \cdots, t_{n+1}, \tau_{1}, \cdots, \tau_{n}$, where $t_{i}$ is a translation in $R^{n}$ one-unit along the $i$-th axis, $1 \leq i \leq n+1$, and $\tau_{i}, 1 \leq i \leq n$, translates one half unit along the $(n+1+i)$ th axis and simultaneously reflects in the $(i, i+1)$ plane.

In coordinate notation,

$$
\begin{aligned}
& t_{i}\left(x_{1}, \cdots, x_{n+1}, y_{1}, \cdots, y_{n}\right)=\left(x_{1}, \cdots, x_{i}+1, x_{i+1}, \cdots, x_{n+1}, y_{1}, \cdots, y_{n}\right), \\
& \quad \tau_{i}\left(x_{1}, \cdots, x_{n+1}, y_{1}, \cdots, y_{n}\right) \\
& \quad=\left(x_{1}, \cdots, x_{i-1},-x_{i},-x_{i+1}, x_{i+1}, \cdots, x_{n} ; y_{1}, \cdots, y_{i}+\frac{1}{2}, \cdots, y_{n}\right) .
\end{aligned}
$$

Note that $t_{1}, \cdots, t_{n+1}$ generate a commutative subgroup which is freely generated by $t_{1}, \cdots, t_{n+1}$. Similarly $\tau_{1}, \cdots, \tau_{n}$ generate a free abelian subgroup of rank $n$. Actually the first subgroup is normal and so we have a (split) exact sequence as follows:

$$
0 \longrightarrow Z^{n+1} \longrightarrow G_{n} \underset{\rho}{\longleftrightarrow} Z^{n} \longrightarrow 1 \text {. }
$$

Surely then it follows that $G_{n}$ is torsion-free. It is clear that $D\left(t_{i}\right)$ is the identity, or equivalently that $t_{i}$ is a translation. While $D\left(\tau_{i}\right)$ is the diagonal matrix with minus ones in rows $i$ and $i+1$ and plus ones elsewhere. Thus the holonomy group $D\left(G_{n}\right)$ is isomorphic to $Z_{2}^{n}$ generated by $D\left(\tau_{i}\right)$. The translations subgroup is the free abelian group generated by $t_{1}, \cdots, t_{n+1}$ and $\tau_{1}^{2}, \cdots, \tau_{n}^{2}$, which, of course, is the standard lattice.

Now, since $M^{2 n+1}=B_{G_{n}}$, we may calculate $H^{i}\left(M^{2 n+1} ; R\right)$ by calculating $H^{i}\left(G_{n}, R\right)$ in the sense of [4]. As remarked previously in $\S 1$ the tangent bundle $\operatorname{map} B_{G_{n}} \stackrel{[\tau]}{\longrightarrow} B_{0(2 n+1)}$ is the composition of a collection of maps induced by homomorphisms. Indeed $[\tau]=\boldsymbol{B}_{i} \circ \boldsymbol{B}_{D}$ where $D: G_{n} \rightarrow$ holonomy is the homomorphism above and $i: D\left(G_{n}\right) \subset 0_{2 n+1}$ is the inclusion map. In this case we can factor each of the homomorphisms further, and that is the basis of the calculation below. 
Proposition 5.1. The Stiefel-Whitney classes $w_{2 i}\left(M^{2 n+1}\right), 0 \leq 2 i \leq n$, are not zero; the others are 0.

Proof. Clearly the homomorphism $D: G_{n} \rightarrow$ holonomy factors through $\rho: G_{n} \rightarrow Z^{n}$, i.e., we may write $D=d \circ \rho$ where $d(0, \cdots, 1, \cdots, 0)=D\left(\tau_{i}\right)$ $i \leq i \leq n$. Now mapping $\rho^{*}: H^{i}\left(Z^{n} ; R\right) \rightarrow H^{i}\left(G_{n}, R\right)$ is one-one because we have a homomorphism $s: Z^{n} \rightarrow G_{n}$ such that $\rho \circ s=I d$ and so $s^{*} \circ \rho^{*}=(I d)^{*}$. Now $H^{*}\left(Z^{n} ; R\right)$ is known, of course, and is the exterior algebra generated by $H^{1}\left(Z^{n}, R\right) \simeq \operatorname{Hom}\left(H_{1}\left(Z^{n}\right), R\right) \cong \operatorname{Hom}\left(Z^{n}, R\right)$. In particular, $H^{i}\left(Z^{n} ; R\right)=0$ if $i>n$. Thus, in particular, $w_{i}(M)=0$ for $i>n$.

Now we have the subgroup $T_{2 n+1}$ of $0(2 m+1)$ consisting of diagonal matrices. Then $T_{2 n+1} \simeq Z_{2}^{2 n+1}$, and clearly $D\left(G_{n}\right) \subset T_{2 n+1}$. Thus the inclusion map $i: D\left(G_{n}\right) \rightarrow 0(2 n+1)$ factors through $T_{2 n+1}$. This is useful because $H^{*}\left(B_{T_{2 n+1}} ; Z_{2}\right)$ is well known and the image of $w_{i}$, the universal Stiefel-Whitney class, in $H^{i}\left(B_{T_{2 n+1}} ; Z_{2}\right)$ is known. Specifically, $H_{1}\left(B_{T_{2 n+1}} ; Z\right)$ is naturally isomorphic to $T_{2 n+1}$ itself, and $H_{1}\left(B_{T_{2 n+1}} ; Z_{2}\right) \simeq H_{1}\left(B_{T_{2 n+1}} ; Z\right) \otimes Z_{2} \simeq T_{2 n+1} \otimes Z_{2}$ $\simeq T_{2 n+1}$. Thus $H^{1}\left(B_{T_{2 n+1}}, Z_{2}\right) \simeq \operatorname{Hom}\left(T_{2 n+1} ; Z_{2}\right), H^{*}\left(B_{T_{2 n+1}} ; Z_{2}\right) \simeq Z_{2}\left[x_{1}, \cdots\right.$, $\left.x_{2 n+1}\right]$ where the $x_{i} \in H^{1}$ correspond to the homomorphism $\varepsilon_{i}: T_{2 n+1} \rightarrow Z_{2}$ :

$$
\varepsilon_{i}(A)= \begin{cases}0 & \text { if } A_{i i}=1 \\ 1 & \text { if } A_{i i}=-1\end{cases}
$$

Now $w_{i}$ corresponds to $\sigma_{i}\left(x_{1}, \cdots, x_{2 n+1}\right) \in Z_{2}\left[x_{1}, \cdots, x_{2 n+1}\right] \simeq H^{*}\left(B_{T_{2 n+1}}, Z_{2}\right)$ where $\sigma_{i}$ is the $i$-th elementary symmetric function; see [15].

Now $D\left(G_{n}\right)$ is in the subgroup $S_{n+1}$ of $T_{2 n+1}$ consisting of matrices having plus ones beyond the $(n+1)$ st row. The inclusion $S_{n+1} \subset T_{2 n+1}$ can readily be calculated. Clearly $H^{*}\left(B_{S_{n+1}} ; Z_{2}\right) \simeq Z_{2}\left[\bar{x}_{1}, \cdots, \bar{x}_{n+1}\right]$ analogously to $H^{*}\left(B_{T_{2 n+1}} ; Z_{2}\right)$, and it is trivial to see that via the inclusion $S_{n+1} \subset T_{2 n+1}$ the elements $x_{i}$ go to $\bar{x}_{i}$ for $1 \leq i \leq n+1$ and $x_{j}$ go to zero for $i>n+1$. Thus $w_{i} \in H^{i}\left(B_{0(2 n+1)}, Z_{2}\right)$ traced back to $H^{i}\left(B_{S_{n+1}}, Z_{2}\right)$ is $\sigma_{i}\left(\bar{x}_{1}, \cdots, \bar{x}_{n+1}\right)$. Now the $\operatorname{map} Z^{n} \stackrel{d}{\longrightarrow} S_{n+1}$ is easily calculated and its effect on $H_{1}\left(Z^{n} ; Z_{2}\right) \rightarrow H_{1}\left(S_{n+1} ; Z_{2}\right)$ is, after the obvious identifications, $d \otimes 1 d: Z^{n} \otimes Z_{2} \leftrightarrow S_{n+1} \otimes Z_{2}$. Thus the dual map $H^{1}\left(B_{S_{n+1}} ; Z_{2}\right) \rightarrow H^{1}\left(Z^{n} ; Z_{2}\right)$ is readily calculated to be

$$
\begin{aligned}
& \bar{x}_{1} \rightarrow z_{1}, \\
& \bar{x}_{2} \rightarrow z_{1}+z_{2}, \\
& \vdots \\
& \bar{x}_{n-1} \rightarrow z_{n-2}+z_{n-1}, \\
& \bar{x}_{n} \rightarrow z_{n-1}+z_{n}, \\
& \bar{x}_{n+1} \rightarrow z_{n},
\end{aligned}
$$

where the $z_{i} \in H^{1}\left(B_{Z^{n}} ; z_{2}\right) \simeq \operatorname{Hom}\left(Z^{n}, Z_{2}\right)$ are the homomorphism $z_{i}\left(a_{i}, \cdots\right.$, $\left.a_{n}\right)=a_{i}(\bmod 2)$.

It is obviously advantageous to use a different basis for $H^{1}\left(Z^{n} ; Z_{2}\right)$; to wit 
$v_{1}=z_{1}, v_{2}=z_{1}+z_{2}, \cdots, v_{n}=z_{n-1}+z_{n}$. Thus in the new basis the map $d^{*}: H^{1}\left(B_{S_{n+1}} ; Z_{2}\right) \rightarrow H^{1}\left(Z^{n} ; Z_{2}\right)$ is $\bar{x}_{i} \rightarrow z_{i}, 1 \leq i \leq n$, and $\bar{x}_{n+1} \rightarrow v_{1}+\cdots$ $+v_{n}$. Thus the universal Stiefel-Whitney class $w_{i} \in H^{i}\left(B_{0(2 n+1)}, Z_{2}\right)$ goes to $\sigma_{i}\left(v_{1}, \cdots, v_{n}, v_{1}+\cdots+v_{n}\right) \in$ Exterior algebra generated by $v_{1}, \cdots, v_{n}$. This is clearly $\sigma_{i}\left(v_{1}, \cdots, v_{n}\right)+\sigma_{i-1}\left(v_{1}, \cdots, v_{n}\right)\left(v_{1}+\cdots+v_{n}\right)$. The lemma below shows that $\sigma_{i-1}\left(v_{1}, \cdots, v_{n}\right)\left(v_{1}+\cdots+v_{n}\right)=i \sigma_{i}\left(v_{1}, \cdots, v_{n}\right)$. Hence $w_{i}$ goes to $(i+1) \sigma_{i}\left(v_{1}, \cdots, v_{n}\right) \in$ Exterior algebra generated by $v_{1}, \cdots, v_{n}=$ $H^{*}\left(Z^{n} ; Z_{2}\right)$. This is clearly zero if and only if $i+1$ is even (in the range $0 \leq i \leq n)$. Hence the proposition is proved.

Lemma 5.2. $\sigma_{i-1}\left(v_{1}, \cdots, v_{n}\right)\left(v_{1}+\cdots+v_{n}\right)=i \sigma_{i}\left(v_{1}, \cdots, v_{n}\right)$.

Proof. Both sides are clearly symmetric elements in $v_{1}, \cdots, v_{n}$, but a look at $H^{i}$ shows that it has a basis consisting of $v_{j_{1}} \cdots v_{j_{i}}, j_{1}<\cdots<j_{i}$.

Now the permutation group clearly permutes this basis transitively; thus the only symmetric elements are 0 , and the element which is the sum of all the basis elements is $\sigma_{i}\left(v_{1}, \cdots, v_{n}\right)$. The lemma follows simply by counting the number of occurences of the monomial $v_{1} \cdots v_{i}$ in $\sigma_{i-1}\left(v_{1}, \cdots, v_{n}\right)\left(v_{1}+\cdots\right.$ $+v_{n}$ ), and this number is clearly $i$.

Corollary 5.3. $n\left(Z_{2}^{2 k}\right) \geq 2 k, \quad n\left(Z_{2}^{2 k+1}\right) \geq 2 k$.

Proof. Combine Proposition 5.1 and Corollary 3.5.

Remarks 5.4. a) If we apply the methods of $\S 4$ we find that the manifold $M^{2 n+1}$ is a flat toral extension of a flat $n$-torus. The splitting of the above sequence is reflected by the existence of a section of this fibration.

b) The fact that $n\left(Z_{2}^{2 k}\right) \geq 2 k$ could be deduced by taking a $k$-fold product of the example of [2] with itself and applying the product formulas for StiefelWhitney classes.

We include now one last example. It also is a sequence of groups having $Z_{2}^{n}$ as holonomy group but in "some sense" which we would not venture to try to make precise is at the opposite extreme from the $G_{n}$ 's.

Definition of $\bar{G}_{2 n}$. The group $\bar{G}_{2 n}$, like the group $G_{n}$, will be a subgroup of $\mathscr{E}_{2 n+1}$ such that the orbit space $R^{2 n+1} / \bar{G}_{2 n}=\bar{M}^{2 n+1}$ is a compact flat Riemannian manifold. Its holonomy group is $Z_{2}^{2 n}$; it will contain $G_{n}$ as a normal subgroup of finite index.

Let $\sigma_{i} \in 0(2 n+1) \subset \mathscr{E}_{2 n+1}$ be reflection in the $i$-th axis, i.e., $\sigma_{i}\left(x_{1}, \cdots, x_{2 n+1}\right)$ $=\left(x_{1}, \cdots,-x_{i}, \cdots, x_{2 n+1}\right), i=1,2, \cdots, 2 n+1$. Let $s_{i}$ be translation by one-half unit in the $i$-th direction, i.e., $s_{i}\left(x_{1}, \cdots, x_{2 n+1}\right)=\left(x_{1}, \cdots, x_{i}+\frac{1}{2}, \cdots\right.$, $\left.x_{2 n+1}\right)$. We note that with this notation the element $\tau_{i}$ above is $\sigma_{i} \sigma_{i+1} s_{i+n+1}$.

Let $Z^{2 n+1}$ be the standard lattice in $R^{2 n+1}$, and $T=R^{2 n+1} / Z^{2 n+1}$ the quotient Clearly $\sigma_{i}$ and $s_{j}$ induce isometries of $T$, which we will denote by $\bar{\sigma}_{i}$ and $\bar{s}_{j}$. Each of these isometries is of order two, and they all commute with one another.

Let $h_{i}=\bar{\sigma}_{i} \bar{\sigma}_{i+1} \bar{s}_{i+n+1}, i=1, \cdots, 2 n+1$. (The subscripts are to be interpreted modulo $2 n+1$.) Let $\Phi_{2 n}$ be the subgroup of isometries of $T$ generated 
by $h_{1}, \cdots, h_{2 n}$. We claim that $\Phi_{2 n}$ is isomorphic to $Z_{2}^{2 n}$ in the obvious fashion and that $\Phi_{2 n}$ acts without fixed point on $T$.

To see this we here really only to show that if $\varepsilon=\left(\varepsilon_{1}, \cdots, \varepsilon_{2 n}\right) \neq(0, \ldots, 0)$ $\in Z_{2}^{2 n}$. Then $h^{\varepsilon}=h_{1}^{\varepsilon_{1}}, \cdots, h_{2 n}^{\varepsilon_{2 n} n}$ has no fixed points in $T$. But surely if $\varepsilon_{i} \neq 0$ we have $h^{e}[x]=[y]$ where $y_{i+n+1}= \pm x_{i+n+1}+\frac{1}{2}$ modulo 1 .

It follows now that $T$ is a regular covering space of the space of orbits $T / \Phi_{2 n} \equiv \bar{M}^{2 n+1}$. Thus $\bar{M}^{2 n+1}$ "is" a compact flat Riemannian manifold. Let $\bar{G}_{2 n}$ denote its fundamental group which we identify with the group of deck transformation of its universal covering space $R^{2 n+1}$. From the covering situation we get an exact sequence of groups

$$
0 \rightarrow Z^{2 n+1} \rightarrow \bar{G}_{2 n} \rightarrow \Phi_{2 n} \rightarrow 1 \text {. }
$$

From this it is clear that $\bar{G}_{2 n}$ is generated by $Z^{2 n+1}$ together with the elements $h_{i}^{\prime}=\sigma_{i} \sigma_{i+1} s_{i+n+1} \in \varepsilon_{2 n+1}, i=1, \cdots, 2 n$. The holonomy group $D\left(\bar{G}_{2 n}\right) \subset$ $0(2 n+1)$ is therefore generated by $\sigma_{i} \sigma_{i+1}, i=1, \cdots, 2 n$, and consequently is isomorphic to $Z_{2}^{2 n}$. Indeed the above sequence "is"

$$
0 \rightarrow t\left(\bar{G}_{2 n}\right) \rightarrow \bar{G}_{2 n} \rightarrow \text { holonomy } \rightarrow 1 .
$$

Now the subgroup generated by $Z^{2 n+1}$ together with $h_{i}^{\prime}, i=1, \cdots, n$, is just the group $G_{n}$ considered earlier. Thus $G_{n}$ is a subgroup of $\bar{G}_{2 n}$. Hence $M^{2 n+1}$ $=R^{2 n+1} / G_{n}$ is a covering space of $\bar{M}^{2 n+1}=R^{2 n+1} / \bar{G}_{2 n}$. As a consequence of 5.1 above, we have proved the following.

Proposition 5.5. $w_{2 i}\left(\bar{G}_{2 n}\right) \neq 0$ for $0 \leq 2 i \leq n$.

Note that unlike Proposition 5.1 we do not assert anything about $w_{i}\left(\bar{G}_{2 n}\right)$ for other values of $i$. Except for the obvious " $w_{1}=0$ " (for $\bar{M}$ is clearly orientable) we know nothing about the others. For $n=1$ however we are dealing with an orientable 3 -manifold which is therefore necessarily parallelizable [11]. Indeed $\bar{G}_{2}$ must be the group $G_{6}$ of [16, pp. 602-603].

\section{References}

[1] L. Auslander \& M. Kuranishi, On the holonomy group of locally Euclidean spaces, Ann. of Math. (2) 65 (1957) 411-415.

[2] L. Auslander \& R. H. Szczarba, Characteristic classes of compact solvmanifolds, Ann. of Math. (2) 76 (1962) 1-8.

[3] L. Bieberbach, Über die Bewegungsgrüppen der Euklideschen Raume. I, II, Math. Ann. 70 (1911) 297-336; 72 (1912) 400-412.

[4] H. Cartan \& S. Eilenberg, Homological algebra, Princeton University Press, Princeton, 1956.

[5] S. S. Cairns, Triangulation of the manifold of class one, Bull. Amer. Math. Soc. 41 (1935) 549-552.

[6] L. Charlap, Flat Riemannian manifolds. I, Ann. of Math. 81 (1965) 15-30.

[7] L. Charlap \& A. T. Vasquez, Compact flat Riemannian manifolds. II, Amer. J. Math. 87 (1965) 551-563.

[8] P. J. Hilton \& S. Wylie, Homology theory, Cambridge University Press, Cambridge, 1960.

[9] S. Kobayashi \& K. Nomizu, Foundations of differential geometry, Interscience, New York, 1963. 
[10] I. Reiner, Integral representations of cyclic groups of prime order, Proc. Amer. Math. Soc. 8 (1957) 142.

[11] N. E. Steenrod, The Topology of fibre bundles, Princeton University Press, Princeton, 1951.

[12] R. Thom, Quelques propriétés globales des variétés différentiables, Comment. Math. Helv. 28 (1954) 17-86.

[13] A. T. Vasquez, On characteristic classes of flat Riemannian manifolds, Bull. Amer. Math. Soc. 74 (1968) 715-717.

[14] C. T. C. Wall, Determination of the cobordism ring, Ann. of Math. (2) 72 (1960) 292-311.

[15] A. Borel, Topology of Lie groups and characteristic classes, Bull. Amer. Math. Soc. 61 (1955) 397-432.

[16] W. Hantzche \& H. Wendt, Dreidimensional Euklidische Raumformen, Math. Ann. 110 (1934-1935) 539-611.

[17] E. Calabi, Closed locally Euclidean four dimensional manifolds, Bull. Amer. Math. 63 (1957) 135.

\section{Graduate Center}

The City University of New York 